\title{
The Current Status of Informatization and Information Literacy in Advanced Medical Institutions
}

\author{
Na Wang ${ }^{1}$ and Jinguo Wang ${ }^{2, *}$ \\ ${ }^{1}$ Department of Anesthesiology, The First Hospital of Jilin University, China \\ ${ }^{2}$ Department of Urology, The First Hospital of Jilin University, China \\ ${ }^{*}$ Corresponding author
}

\begin{abstract}
This paper analyzes the main problems and causes in the practice of information quality education in higher medical institutions. The paper puts forward the effective way to cultivate and improve the information quality of medical students by the education curriculum and teaching. Mainly Though the build scientific and rational curriculum system of medical students information literacy, the integration of information quality education and the medical professional courses and quality evaluation standard system, teachers' and students' information literacy are improved. The role of libraries of the advanced medical colleges and universities must be given full play. The cooperation of librarians and medical professional teachers must be reasonably strengthened to create a good environment for campus informatization to promote development of the information quality education and improve the information literacy of medical students and teachers.
\end{abstract}

Keywords-informatization; information literacy; medical science

\section{INTRODUCTION}

The medical science is the fastest field of development. The progress of medical technology, medical information and medical knowledge update at a amazing speed. The cycle of advancement speeds up unceasingly. The information technology is widely used in medicine. The information-based degree of clinical medical work is more and more high. In the future, doctors' work faces a growing and increasing complex area of the clinical diagnosis and treatment work. It endows with higher demand and more responsibility. The importance of information quality will become the clinical medical work conditions and the necessary quality in the future. [1]

Training qualified information talents of advanced medical education for the society is facing the severe challenges in the information age. The advanced medical colleges and universities which cultivate medical graduates have concrete medical professional knowledge and good clinical skills. At the same time, they should also cultivate their good information literacy. Information quality education is an important issue in advanced medical colleges and universities.

\section{CONCEPT OF INFORMATION LITERACY}

\section{A. Information Awareness Quality}

Information consciousness refers to the information in people's minds which reflect the qualification of the career. People have sensibility and sustained attention to information and they have judgment and insight of information value. [2]

\section{B. Information Ability Quality}

Information ability refers to the ability of people to obtain, process and use information. It has become a problem that everyone must face how to extract useful information from the vast information resources. Information ability is one of the skills that people must possess in a new age. [3]

\section{Information Moral Quality}

Information ethics refers to the moral norms that people should follow in the information activities, such as protecting intellectual property rights, respecting privacy and resisting bad information. The network has a great negative effect, as well as an endless benefit of our life.

The network space is a mixture. The infringement of copyright behavior is increasingly rampant and the information environment is being seriously polluted, so training the correct information channel is becoming more and more important. [4]

\section{HistORY OF THE CULTIVATION OF INFORMATION LITERACY}

The research on domestic information capacity was originated in the early 1980s. As our country began to clean up the far-left thoughts influence in the fields of social economy, science and culture, academic research is broken gradually, inspired by the situation of many scholars, starting from the perspective of science and technology. The economic and cultural development actively brings up their respective discipline. [5]

Some scholars have proposed to develop the scientific capacity of our country. When they deal with the basic content of social science, the efficiency of the digital intelligence system is also an important aspect. Some scholars in the book 
intelligence community have been enlightened and began to discuss the problem of information ability in China.

Domestic scholars generally use intelligence to characterize the information we use today. Accordingly, researchers generally use intelligence with a little information capability. After many years unavoidably in the blind state of information, researchers noticed that the intelligence ability of student learning, scientific research and enterprise management are important. Emphasis on intelligence ability is one of the most basic professional skills or creativity structure elements. Emphasis of the library organization to carry out information education improves the ability of users' information. [6]

The primary index of the evaluation index system is usually the index of sub-index or criterion property, which is the refinement of the total target. Generally, the primary index cannot be judged directly, but it should be evaluated according to the standard of the underlying index.

Though the information ability is a dynamic concept, in different stages it has different connotation and denotation. Both at home and abroad, it has not yet been a unified definition and it includes the study of individual and the whole society. This article refers to information ability, which refers to the individual user information ability.

A person with information ability, information knowledge should be followed by the ability to use computer to collect information. The information ability is the ability of processing, analyzing and identifying information.

\section{OVERVIEW OF FOREIGN INFORMATION CAPABILITY RESEARCH}

In the United States, information capabilities have long been incorporated into the national education goals. The training of information capabilities has been incorporated into the curriculum. Researchers from all disciplines and in various social production departments have begun to study the information capabilities of their respective disciplines of their departments. More and more scholars are concerned about the performance of information ability in various work stations, the mechanism of function and the way of cultivation.

There are numerous articles on case studies and theoretical studies of information capability. Governments are also actively trying to improve the information capabilities of countries and citizens. The current research areas include several aspects, including how to calibrate the level of information ability and the degree of progress, how to measure information ability training program to improve individual quality effect, how for information to play a role in different working environments and how to improve labor productivity. [7]

\section{DOMESTIC INFORMATION CAPABILITY RESEARCH}

Most of the domestic researchers take information ability as information retrieval, information processing ability, information analysis ability, as well as library using ability. However, in the 1980s, the researchers emphasized the cultivation and improvement of information ability. Especially for the librarians and college students' information ability training method of the research is very active. They emphasized the significance of the literature retrieval course in colleges and universities. The various units of its own opinions are on the specific situation. [8] They not only carried out all kinds of specialized subjects, such as science and technology workers, farmers, businessmen, journal editors, the composition of the graduate students and teachers' information ability cultivation approaches of research, but also carried out subject information users on a general sense. Libraries and achievement improve the way of research.

Domestic information research ability on the content of a distinctive feature is the introduction of foreign literature achievements and progress and the basic way is to compile or review on foreign related research literature. The current research focuses on the definition, main content, main evaluation methods and teaching practice of some schools. Also some scholars pay attention to the research content of localization, the analysis of the similar foreign research under the background of theoretical framework and practical experience. Quality education activities of domestic college students information has been carried out. The research and research fields are still hot.

\section{CHINESE EVALUATION STANDARD RESEARCH}

It is not difficult to find that it is based on the definition of information quality of the American library association. From the formation of information database, the required information acquisition, assessment of obtained information, the use of information and related economic, legal and social responsibility, etc., this paper also expounds the essential skills in the process of information collection and utilization.

It focuses on the assessment of information ability and information ethics. At present, domestic scholars have formed a unified understanding of the content system of information literacy. That includes three parts, information awareness, information ability and information ethics. Therefore, it is not enough to evaluate Chinese information quality education with American standard.

The evaluation indexes of information awareness and other aspects need to be supplemented. It has been a long time since Chinese colleges and universities carried out information quality education. Most of the papers remain on the technical aspects of how literature retrieval courses to be opened and taught. There are not many papers on the information quality education.

The evaluation standard of education of information quality in China reflects the advantages of network, database, multimedia and other information technologies for users to collect and utilize information. The content of information ability, information awareness and information morality are evaluated. [9]

The specific content includes the content and scope of the specific information needs, the efficient access to the required information, the information evaluation and information sources to assess its value and effectiveness. It integrates the acquired information into your own knowledge system and use 
information to accomplish specific tasks. The economic, political, legal and social norms associated with information use.

\section{TRAINING OBJECTIVES OF INFORMATION LITERACY}

The training objectives of information literacy include several aspects. How to choose and judge information sources is an important aspect. The advantages and disadvantages of the alternative information must be judged. How to master the ability to use various information from different counties is necessary. How to use the relevant information caves to improve the project review and evaluation is needed. Information is a resource and has value of wealth.

Because the database has great storage capacity and low price, simple and convenient method of use, it can solve the printed retrieval tool and has the advantages such as aging. It makes the disc play a more and more important role in information retrieval. It can be used both on single disk and on line. [10]

Many university libraries have to purchase the database for information retrieval, so they want to introduce students to the databases which can let they know what they will need to check, what kind of information in the database, how to check questions and so on. For databases abroad, because of its instruction, methods are used when retrieving methods similar to those of international online retrieval systems, it introduced the online retrieval. The key is to introduce the domestic several databases.

\section{SEtTing UP A FUll-Time TeAChing StafF}

The teaching of information quality is mainly for the teacher and the library staff or information management department. This kind of staff teaching structure has its unreasonable aspect. It should build a group that combines the professional teacher with the quality teacher troop to apply their respective specialty.

Online information scattered disorderly, but its distribution has a general rule with timeliness. It has four types of information including electronic journals, electronic newspapers, dynamic information and bibliographic database. If it is divided by form, it also includes text information and non-text information. If it is divided according to the processing degree, it can include the original information and index guide. [11]

Its original sources of information are similar. But due to the particularity of the network transmission, there are specially applicable to the distribution of the information network. It should include the following categories, including Book information, meeting information, patent information, government publications information, information provided by universities, information provided by commercial companies, research institutions and academic groups. Information contains in the newsgroup and communication discussion groups, information provided by libraries on the Internet, online information provided by the commercial online retrieval system. There is also information provided by professional information service providers.
It's not just about the types of information you need to introduce, but also about what information services are available in each type and which sites can get the information you need most in.

\section{SUMMARY}

The determination of information literacy of college students requires a relative standard or accepted definition of information literacy. The concept of information literacy is not unified without international standard definition, so we used the foreign reference extensively. Relevant authorities or organizations would agree with the definition of information literacy made by the American library association. It is namely that the information literacy is to realize that when you need information, you have the ability to find, evaluate and effectively use the information needed.

In a fundamental sense, people with information literacy are those who know how to learn. They know how to learn, because they know how to organize the knowledge, how to find information and how to use information, so that others can learn from them, and they have been ready for lifelong learning.

\section{REFERENCES}

[1] Bimber. Three aces of Technological Determinism. Does Technology Drive History?: The Dilemma of Technological determinism. 1994

[2] Burrows S. Developing an "evidence-based medicine and use of the biomedical literature" component as a longitudinal theme of an outcomes-based medical school curriculum: year 1. J Med LibrAssoc. 2003

[3] John Weckert. What is so bad about Internet content regulation?[J]. Ethics and Information Technology. 2000 (2)

[4] Tomas A. Lipinski, Johannes Britz. Rethinking the ownership of information in the 21st century: Ethical implications[J]. Ethics and Information Technology. 2000 (1)

[5] Joseph D Bronzino. Health Care and Information Ethics:Protecting Fundamental Human Rights. Health Progress. 1999

[6] Herman T. Tavani. The state of computer ethics as a philosophical field of inquiry: Some contemporary perspectives, future projections, and current resources[J]. Ethics and Information Technology. 2001 (2)

[7] Alison Adam,Jacqueline Ofori-Amanfo. Does gender matter in computer ethics?[J]. Ethics and Information Technology . 2000 (1)

[8] Tavani. H.T. The State of Computer Ethics As A Philo-sophical Field of Inquiry:So me Contemporary Perspec-tives,Future Projections, and Current Resourcs. Ethics and Information Technology. 2001

[9] Lipinsld T A, Britz J. Rethinking the Ownership of Information in the 21stCentury: EthicalImplications. Ethics and Inform ation Technology. 2000

[10] Tim Jordan. Cyberpower-the culture politics of cyberspace and the internet. 1999

[11] Thompson P.B. Privacy, Secrecy and Security. Ethics and Information Technology. 2001 